\title{
Breast Abscesses: Diagnosis, Treatment and Outcome
}

\author{
Markus Fahrni ${ }^{a} \quad$ Esther I. Schwarz ${ }^{c}$ Sylvia Stadlmann ${ }^{b} \quad$ Gad Singer $^{b}$ \\ Nik Hauser ${ }^{c}$ Rahel A. Kubik-Huch ${ }^{a}$ \\ anstitute of Radiology, \\ bInstitute of Pathology, \\ 'Department of Obstetrics and Gynecology, Kantonsspital Baden AG, Baden, Switzerland
}

\author{
Keywords \\ Breast - Abscess - Ultrasonography . \\ Fine needle aspiration - Drainage
}

\section{Summary}

Background: The aim of our study was to analyze diagnostic results, different treatment modalities, and the outcome of patients with breast abscesses treated at our institution in a multi-modality breast team, to determine whether minimally invasive treatments are successful. Methods: 110 patients with mastitis and suspected breast abscesses at our institution between January 2000 and end of September 2007 were retrospectively analyzed. Abscesses were diagnosed using ultrasonography (US), and the material obtained using US-guided fine needle aspiration (FNA) was further examined. Results: $29 \%$ of the patients were treated conservatively with antibiotics only, $51 \%$ were treated with US-guided FNA or drainage placement. $11 \%$ of the patients underwent additional surgery after minimally invasive treatment (i.e. conversion rate). $9 \%$ of the patients underwent primary surgery. Early complications occurred in $7 \%$ of patients treated minimally invasive but not in patients treated with surgery alone. Late complications occurred in $5 \%$ of patients who underwent minimally invasive treatments and in $30 \%$ of patients who underwent surgery. Conclusions: US-guided FNA as a minimally invasive therapy in combination with antibiotics was found to successfully treat most breast abscesses and, in cases where a larger volume of pus was involved, the placement of an additional drainage catheter was effective.

\author{
Schlüsselwörter \\ Brust · Abszess · Ultraschall · Feinnadelaspiration · \\ Drainage
}

\section{Zusammenfassung}

Hintergrund: Ziel unserer Studie war es, die diagnostischen Ergebnisse, die verschiedenen Therapiemodalitäten und das Behandlungsergebnis von Patienten und Patientinnen mit Brustabszessen, die durch das interdisziplinäre Brustteam unserer Institution behandelt wurden, zu analysieren und herauszufinden, ob das minimal invasive Therapieschema erfolgreich ist. Methoden: Die Daten von 110 Patienten mit Mastitis und Verdacht auf Abszessbildung, die zwischen Januar 2000 und September 2007 in unserer Institution behandelt wurden, wurden retrospektiv ausgewertet. Die Abszesse wurden mittels Ultraschall (US) diagnostiziert, und das mittels US-gesteuerter Feinnadelaspiration (FNA) gewonnene Material wurde weiter analysiert. Ergebnisse: 29\% der Patienten wurden konservativ mit Antibiotika behandelt, bei $51 \%$ wurde zusätzliche eine US-gestützte FNA oder Drainageeinlage durchgeführt. $11 \%$ der Patienten wurden nach der US-gestützten Intervention operiert (= Konversionsrate). 9\% der Patienten wurden primär operiert. Frühkomplikationen traten bei $7 \%$ der minimal invasiv behandelten und bei keinem der primär operierten Patienten auf. Spätkomplikationen wurden hingegen bei $5 \%$ der minimal invasiv behandelten und 30\% der primär operierten Patienten beobachtet. Schlussfolgerungen: Die minimal invasive US-gestützte FNA ist in Kombination mit Antibiotikagabe bei der Behandlung von Brustabszessen in den meisten Fällen erfolgreich. Bei großen Abszessen empfiehlt sich die ergänzende Einlage eines Drainagekatheters.

\section{KARGER \\ Fax +497614520714 \\ Information@Karger.de}

www.karger.com
(C) 2012 S. Karger GmbH, Freiburg

1661-3791/12/0071-0032\$38.00/0

Accessible online at:

www.karger.com/brc
Prof. Dr. med. Rahel Kubik-Huch, MPH

Institute of Radiology

Kantonsspital Baden AG

5404 Baden, Switzerland

Tel. +41 56486 38-02, Fax -09

rahel.kubik@ksb.ch 


\section{Introduction}

Breast abscesses are a complication of mastitis, and a pyogenic abscess can evolve from acute bacterial mastitis if treatment with antibiotics is not successful. Breast abscesses are more frequently observed in non-puerperal mastitis than in puerperal mastitis, and can be a particularly difficult condition due to the intense discomfort and tendency for recurrence. When an abscess is small and located deep within the breast, it can be clinically difficult to detect and differentiate from mastitis; therefore, ultrasonography (US) is the method of choice for diagnosis [1,2].

Breast abscesses have traditionally required a surgical incision to allow drainage of the abscess, which is usually performed under general anesthesia followed by administration of antibiotics [3]. However, this treatment strategy can interfere with lactation and potentially yields poor cosmetic results. More recently, the use of US-guided needle aspiration, or drainage with a catheter, has been shown to successfully treat breast abscesses [4]. In this study, the aim was to retrospectively analyze diagnostic results, different treatment modalities, and the outcome of patients with breast abscesses treated at our institution in a multi-modality breast team, to determine whether minimally invasive treatments are successful.

\section{Materials and Methods}

\section{Patient Selection}

A total of 110 patients (107 women, 3 men; average age 40 years; age range 14-82 years) with mastitis and suspected breast abscesses were treated at the Institute of Radiology and the Department of Gynecology/Senology/Obstetrics. This breast center is certified according to ISO 9001:2000, as well as by the 'Deutsche Gesellschaft für Senologie' (German Society for Senology) and the 'Deutsche Krebsgesellschaft'

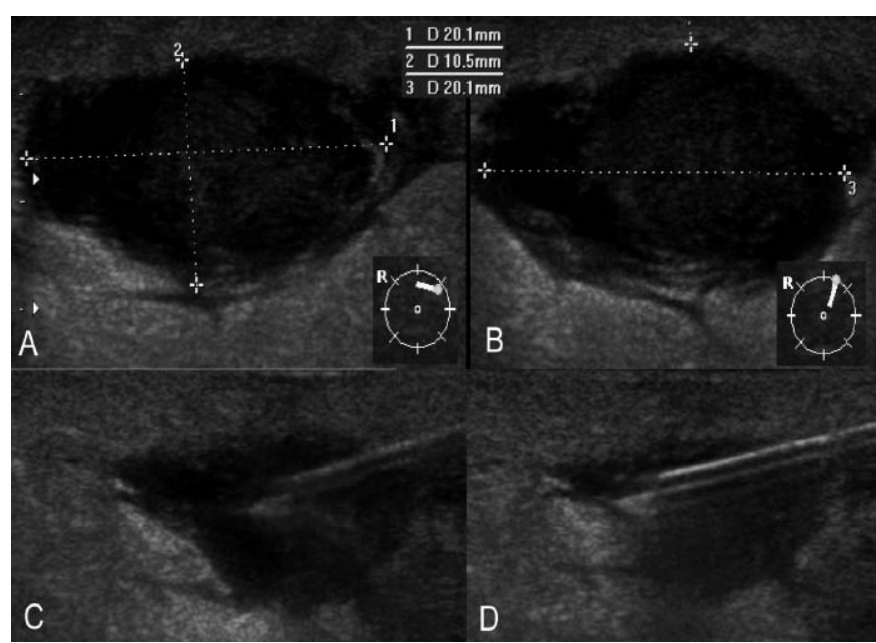

Fig. 1. Ultrasound images of a breast abscess and US-guided FNA in a woman of 53 years. (A) Shows axial US image of a large hypoechogenic lesion (collection of pus) with perifocal hyperechogenic tissue;

(B) shows the corresponding longitudinal image; (C, D) following USguided insertion of a needle, the material of the abscess is aspirated.
(German Cancer Society), and patients had treatment and imaging available to them as determined to be necessary by a multi-modality breast team. This retrospective study was approved by the Institutional Review Board of this institution.

All patients diagnosed with breast abscesses and/or localized acute inflammation of the breast between January 2000 through September 2007 were identified using the Radiology Information System (RIS) Conrad (Cobrasoftware AG, Arlesheim, BL, Switzerland) or the Picture Archiving and Communication System (PACS) Centricity 4.1 (GE, Milwaukee, WI, USA). Further patient information relevant to the study, e.g. risk factors for developing breast abscesses and therapeutic information as described in the Data Analysis section below, was retrieved from gynecological case histories. Data from external radiological examinations and from microbiological and cytopathological assays were also obtained. Finally, all patients whose case history and course of disease were documented for at least 6 months were included in this study. Patient data were analyzed using the statistics tools of Excel (Microsoft Corp., Redmond, WA, USA).

\section{Data Analysis}

The following data were analyzed: (1) patient clinical history, including risk factors and diagnostic clarifications (i.e. radiological imaging, microbiology and cytopathology assay data); (2) types of therapy administered (conservative, minimally invasive, and primary surgery); (3) treatment outcome.

Nicotine abuse, diabetes mellitus (DM), fibrocystic changes, or ipsilateral surgical breast interventions combined with previous radiotherapy were evaluated as possible significant, predisposing risk factors for breast abscesses in our patient pool.

In the Outcome section, the early and late complication rates, number of recurrences, and cases involving a change in treatment strategy (i.e. the change from invasive to surgical therapy due to progression of the disease, the so-called conversion rate) are summarized. Early complications were defined as post-operative hematoma, post-operative seroma, or post-interventional technical complications (i.e. the obliteration of a drainage catheter). Late complications included increased scarring events (including parenchyma defects following incision and mammillary retraction) and the formation of a galactocele. The incidence of recurrence was evaluated independently from late complications, and was defined as a new abscess or localized acute inflammation of the same breast within 3 months to 4 years from the completion of therapy, which was defined as the remission of clinical symptoms and completion of all therapeutic procedures, including antibiotic therapy.

\section{Techniques}

\section{Minimally Invasive Therapy}

Minimally invasive treatment of breast abscesses was performed using US-guided fine needle aspiration (FNA), or by US-guided percutaneous drainage placement. For US-guided FNA, superficial disinfection and local anesthesia were administered before a 20-gauge needle, or a 22gauge needle with a mandrin, was used to aspirate the center of the abscess (fig. 1). For viscous contents, an 18-gauge needle was used, and where aspiration was difficult, the abscess cavity was flushed with sterile saline solution.

For drainage placement, US-guided FNA was performed followed by percutaneous insertion of a standard drainage catheter, mostly 7 French, by prick incision (fig. 2). Alternatively, a drainage catheter was inserted directly as guided by US, from which samples could be aspirated.

\section{Primary Surgery}

Surgical management of a breast abscess involved the administration of general anesthesia before an incision was made to completely open the 


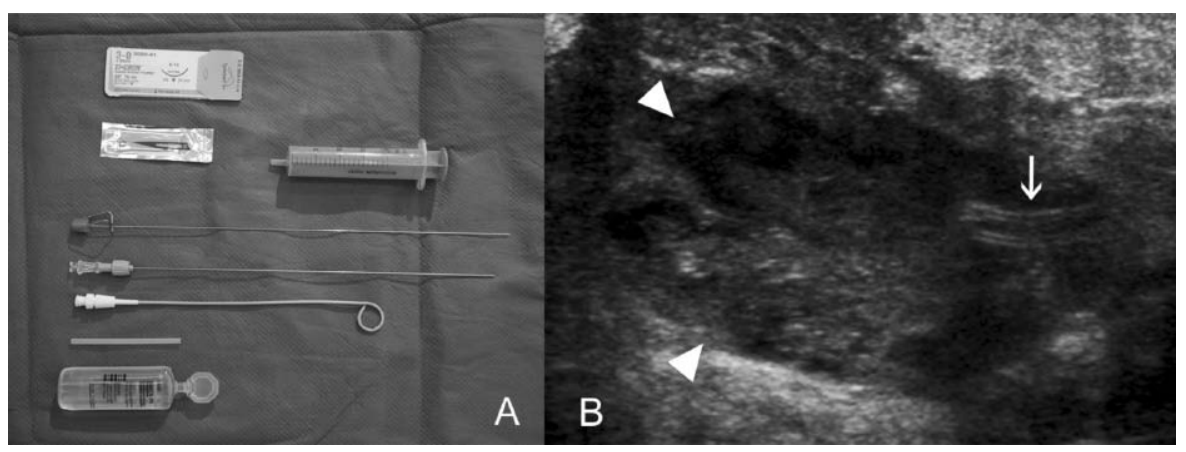

Fig. 2. US-guided drainage of an abscess formation in a 28 -year-old patient. (A, B) Shows the tools used for drainage of an abscess and the catheter tip (marked with an arrow) in the abscess cavity (indicated with short arrows).

abscess cavity. A microbiological smear and resection of a tissue sample from the abscess cavity was performed before the abscess cavity was extensively irrigated, necrotic tissue removed, and a large-lumen drainage catheter inserted. Post-operatively, the wound cavity was kept open by drainage and was irrigated at regular intervals. Typically, abscesses larger than $3 \mathrm{~cm}$ in diameter were treated by US-guided drainage or surgical incision. However, other clinical parameters, i.e. localization and surrounding phlegmonous inflammation, were also considered in the selection of treatment. The treatment options available to each patient were discussed, and the patient's preference was taken into consideration for the final decision regarding treatment.

\section{Results}

\section{Clinicopathological Results}

The mean age of the 107 women with breast abscesses included in this study was 40 years (range: 14-82) and they experienced the following clinical forms of inflammation: non-puerperal mastitis $(57 / 107,53 \%)$, puerperal mastitis $(34 / 107,32 \%)$, or other forms of inflammation (e.g. folliculitides or infected seromas) (16/107, 15\%).

Three men with non-puerperal mastitis were also included in this study and had a mean age of 47 years (range: 42-53). 1 male patient (46 years old) described a breast abscess experienced 1 year earlier; however, no medical records were available for this event. Therefore, with only the patient's own description of this first inflammatory process, the breast abscess that presented during this study was not classified as a recurrence. Another male patient (53 years old) presented with a retroareolar abscess, and gynecomastia was found to be present.

\section{Risk Factors}

Overall, fibrocystic changes were detected in $39 \%$ of the cohort (43/110). In cases of non-puerperal mastitis, $33 / 57$ (58\%) patients presented with fibrocystic changes, in cases of puerperal mastitis, 3/34 (9\%) presented with fibrocystic changes, and in cases of other forms of inflammation, 7/16 (43\%) were associated with fibrocystic changes. A status following breastpreserving surgical therapy with radiotherapy because of a carcinoma was present in $6 / 110(5 \%)$ patients. All 6 of these patients were cases of 'other forms of inflammation' (6/16, $38 \%$ ). Additionally, the risk factor of DM was documented in only $3 \%$ of cases, and for $35 \%$ of the total cases, no information regarding DM status was available.

\section{Diagnostic Results}

US was used to diagnose breast abscesses in 109/110 (99\%) patients, with 1 patient undergoing dynamic contrast-enhanced magnetic resonance imaging (MRI) of the breast for diagnosis. Of the patients initially examined by US, 4/109 (4\%) underwent additional breast MRI (fig. 3). The mean size of the abscesses detected was $21 \times 27 \mathrm{~mm}$, and ranged from $4 \times 5 \mathrm{~mm}$ to $57 \times 90 \mathrm{~mm}$.

When minimally invasive treatment of breast abscesses was performed using US-guided FNA, or with US-guided percutaneous drainage placement, 67/109 (61\%) patients had the obtained material examined microbiologically, and for 64 cases (58\%), cytopathological diagnosis of the collected material was performed. Of the FNA samples, $46 / 67$ (69\%) showed growth in bacterial cultures, with $38 / 46(83 \%)$ producing a monoculture, and 8/46 (17\%) producing a mixed culture. Table 1 lists the pathogens identified in these 67 cases, and due to the detection of mixed cultures, the overall number (n) of pathogens identified is higher than the number of samples obtained. Blood cultures were not analyzed in this study. As an additional finding, a 62-year-old postmenopausal woman was diagnosed with invasive ductal carcinoma of the breast (fig. 4B), and no growth was detected in a bacteriological assay of this sample.

In the majority of cases $(64 / 110,58 \%)$, a sufficient amount of material collected from FNA could be analyzed by both microbiological and cytopathological assays. During the cytopathological analysis, the aspirates were observed to contain yellow purulent material. In thick cellular smears, acute inflammation could be observed with and without fat necrosis, macrophages, multinucleated giant cells, and cell debris (fig. 4A). For retroareolar abscess material, the aspirate was dominated by suppurative granulomatous inflammation due to the release of squamous material from the ducts into the 


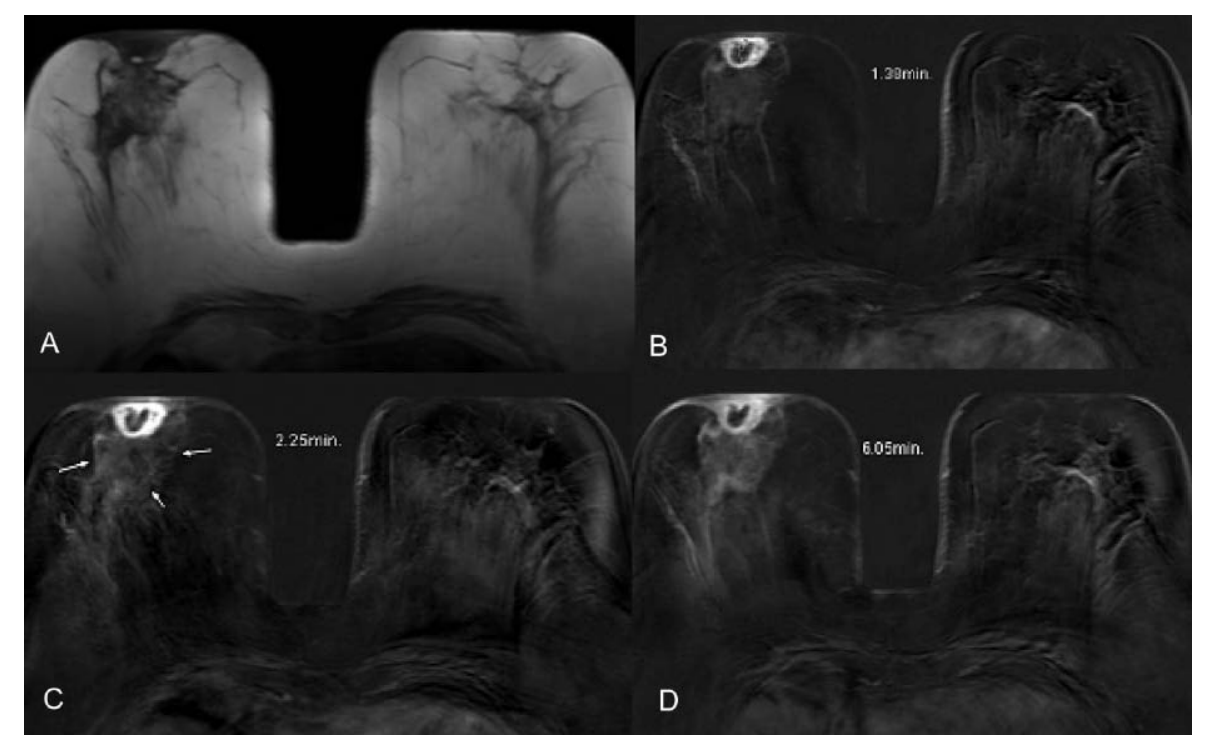

Fig. 3. Contrast-enhanced MRI scans of a retroareolar abscess in the right breast of a 70-year-old patient with non-puerperal mastitis. (A) Shows the T2-weighted axial sequence; (B-D) dynamic contrast-enhanced T1-weighted MRI sequences (subtracted images) with the retroareolar florid abscess noticeably demarcated. A perifocal zone of diffuse inflammatory enhancement is marked with small arrows.

Table 1. Spectrum of pathogens associated with different types of breast inflammation

\begin{tabular}{lrr}
\hline Pathogen & $\mathrm{n}$ & $\%$ \\
\hline Pathogens detected in non-puerperal mastitis cases & 47 & \\
No growth & 14 & 30.0 \\
Staphylococcus aureus & 8 & 17.0 \\
Skin flora & 5 & 11.0 \\
Coagulase-negative staphylococci & 4 & 9.0 \\
Prevotella spp. & 4 & 9.0 \\
Proteus mirabilis & 3 & 2.2 \\
Corynebacterium species & 3 & 2.2 \\
Peptostreptococcus spp. & 1 & 0.7 \\
Staphylococcus lugdunensis & 1 & 0.7 \\
Cellulomonas spp. & 1 & 0.7 \\
Anaerobic gram-negative bacilli & 1 & 0.7 \\
Actinomyces israelii & 1 & 0.7 \\
Candida albicans & 1 & 0.7 \\
Pathogens detected in puerperal mastitis cases & 24 & \\
No growth & 3 & 13.0 \\
Staphylococcus aureus & 19 & 79.0 \\
Coagulase-negative staphylococci & 1 & 4.3 \\
Comamonas acidovorans & 1 & 4.3 \\
Pathogens detected in other inflammation cases & 7 & \\
No growth & 4 & 57 \\
Staphylococcus aureus & 2 & 29 \\
Greening streptococci & 1 & 14 \\
\hline
\end{tabular}

stroma. In such cases, giant cells were found to engulf the squames present. Epithelial and mesenchymal cells with inflammatory or reparative atypia were also sometimes present, mimicking malignancy.

In a 62-year-old postmenopausal woman, as a secondary finding, an invasive ductal carcinoma of the breast was diagnosed (fig. 4B). The bacteriological examination of this sample did not show germ growth.

\section{Treatment}

For 32/110 (29\%) patients, antibiotics alone were prescribed as a conservative treatment; however, some of the patients who underwent minimally invasive techniques or surgery also received antibiotics. For therapies involving antibiotics alone, or as an adjuvant therapy $(35 / 54,65 \%)$, a combined broadspectrum penicillin and $\beta$-lactamase inhibitor, amoxicillin/clavulanic acid, was prescribed. In an additional 18/54 (33\%) cases, the penicillinase-resistant penicillin, flucloxacillin, was prescribed. Occasionally, other antimicrobial agents such as macrolides, cephalosporins, lincosamides, or quinolones were prescribed. Minimally invasive techniques to treat breast abscesses included US-guided FNA $(\mathrm{n}=48)$ and US-guided drainage placement $(\mathrm{n}=8)$. For US-guided FNA, $43(90 \%)$ cases required a single aspiration, $4(8 \%)$ cases required 2 aspirations within a short period, and $1(2 \%)$ case required 4 aspirations within a short period. The additional placement of a drainage catheter following aspiration was the decision of the attending physician, and the reasons for these placements were not available for these cases. The third type of treatment administered for breast abscesses was primary surgery and $10 / 110(9 \%)$ patients underwent this procedure.

\section{Outcome}

\section{Early Complications}

In $4 / 56(7 \%)$ patients who were treated with minimally invasive techniques, early complications were experienced (table 2 ). In the first case, the cavity remaining from a seroma following aspiration of an abscess kept refilling for 2 days. It was successfully treated with 4 aspirations within the course of treatment. In the second case, where a patient received a drainage placement, a hematoma occurred at the insertion point of the catheter and it persisted over 3 days. In the third case, the catheter used during placement of a drainage was occluded after 2 days. Subsequently, the patient was successfully treated surgically. In the fourth case, the abscess ulcerated 

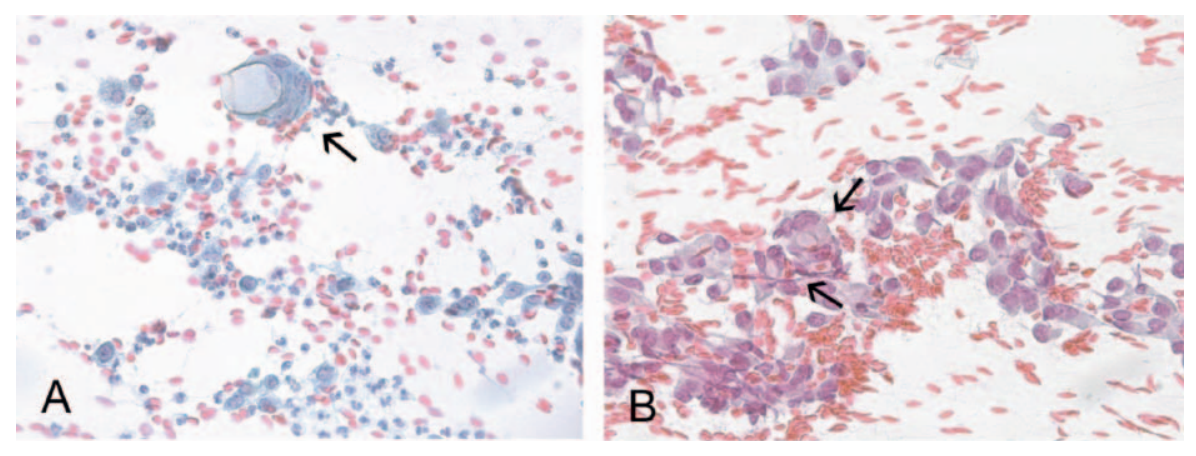

\begin{tabular}{|c|c|c|c|c|c|}
\hline & \multirow[t]{2}{*}{ Patients } & \multicolumn{2}{|c|}{ Complications } & \multirow[t]{2}{*}{ Recurrent disease } & \multirow[t]{2}{*}{ Conversior } \\
\hline & & Early & Late & & \\
\hline & $\mathrm{n}(\%)$ & $\mathrm{n}(\%)$ & $\mathrm{n}(\%)$ & $\mathrm{n}(\%)$ & $\mathrm{n}(\%)$ \\
\hline Conservative therapy & $32(29)$ & $0(0)$ & $0(0)$ & $4(13)$ & \\
\hline Minimally invasive therapy & $56(51)$ & $4(7)$ & $3(5)$ & $5(9)$ & $12(21)$ \\
\hline US-guided FNA & $48(44)$ & $2(4)$ & $1(2)$ & $3(6)$ & $6(13)$ \\
\hline US-guided drainage placement & $8(7)$ & $2(25)$ & $2(25)$ & $2(25)$ & $6(75)$ \\
\hline Surgery & $22(20)$ & $0(0)$ & $3(14)$ & $1(5)$ & \\
\hline Primary surgery & $10(9)$ & $0(0)$ & $3(30)$ & $1(10)$ & \\
\hline Second surgery (conversion) & $12(11)$ & & & & \\
\hline
\end{tabular}

Fig. 4. Papanicolou staining of a cytologic smear from fine needle aspirates from a 61-year-old woman and a 63-year-old woman shows (A) acute inflammation with numerous neutrophil granulocytes, scattered macrophages, and cell debris in the background. Multinucleated giant cells engulfing necrotic fat cells are marked with an arrow (magnification, $20 \times$ ). (B) A homogeneous population of poorly cohesive cells with nuclear atypia, with intracytoplasmic vacuoles present due to mucin production (indicated with arrows) (magnification, $40 \times$ ).

Table 2. Treatment and outcome of patients with breast abscess $(n=110)$ through the skin surface 1 day after US-guided FNA was performed. This patient was also successfully treated with surgery. In contrast to these patients who received minimally invasive treatments, patients who underwent surgery as a primary treatment did not experience any early complications.

\section{Late Complications}

No late complications were experienced by patients treated conservatively.

In $3 / 56(5 \%)$ patients who were treated with minimally invasive procedures, late complications occurred (table 2). For example, a patient treated with US-guided FNA experienced a therapy-resistant inflammation of the breast, which had to be treated with breast ablation. In 2 other cases where patients were treated with US-guided drainage placement, a galactocele formed in each of the previously drained abscess cavities. In one case, the galactocele was successfully treated with a 1-time US-guided FNA, and in the other case the galactocele was successfully treated surgically.

For patients who underwent surgery as a primary treatment, late complications were reported in 3/10 (30\%) cases (table 2). In the first case, a hardened, hypertrophic scar caused discomfort for the patient in the area of the incision. In the second case, a cosmetic scar that caused discomfort developed within the area of the surgery and caused a parenchyma defect. In the third case, large amounts of pus were discharged from the surgical wound 4 weeks after surgical therapy.

\section{Recurrent Disease}

In 10/110 (9\%) patients, a recurrent abscess occurred between 4 months and 4 years following the completion of therapy (table 2). 4 of these patients were part of the conservatively treated group, with 3 of the patients receiving additional antibiotics to treat the recurrence, and 1 patient undergoing surgery to treat the recurrence. For the remaining 5 patients, they were initially treated with minimally invasive techniques before the abscess recurrence occurred. 3 of these 5 patients were treated using US-guided FNA, and 1 of these 3 patients was successfully treated with antibiotics, while the other 2 cases were treated with surgery. For the 2 patients who initially received US-guided drainage placement, recurrences were successfully treated with surgery. In contrast, 1/10 patients who were treated primarily with surgery experienced a recurrence that was successfully treated with antibiotics.

\section{Conversion}

For 6 patients who underwent US-guided FNA and 6 patients who received percutaneous drainage placement, early or late complications, or recurrences, occurred and these patients then needed to undergo surgery as well. Therefore, a conversion rate of $11 \%(12 / 110)$ was associated with this study.

\section{Discussion}

The results of this study show that US-guided therapy of breast abscesses, in combination with antibiotics, is a minimally invasive treatment strategy that can replace surgery for the successful treatment of most cases of breast abscess, and is consistent with the results of other studies [5-8]. For lactating women with puerperal mastitis, the smaller incision site associated with minimally invasive therapy allows them to resume nursing earlier, reduces the possibility for the occurrence of 
galactostasis and is associated with a positive psychological effect [9]. While aspiration alone was used to treat abscesses smaller than $3 \mathrm{~cm}$, larger and uncapsulated abscesses typically required the insertion of a drainage catheter. This was performed for $8 / 56(14 \%)$ of the patients in this study, a rate that is consistent with that reported in other studies $[8,9]$. Most of the patients who received drainage placements were able to experience the same advantages as provided by US-guided FNA: better cosmetic results, no need for general anesthesia, a shorter duration of hospitalization, and lower overall treatment costs. In addition, an advantage of drainage placement versus FNA alone is the ability to rapidly evacuate larger abscesses and their viscous contents. However, a key disadvantage of catheter placement compared to aspiration alone is that, in complex abscess formations, e.g., multi-septated abscesses, the drainage may not access all of the individual compartments present, thereby preventing a complete and rapid drainage. This disadvantage, however, is balanced by the consideration that, upon placement of the drainage, individual septa of the abscess can tear during the process of catheter insertion, and thus a similar effect to the use of surgical instruments or manual 'breaking' of the septa can be achieved. Additional disadvantages associated with the placement of a drainage using a thicker catheter (7 French) are a potentially more painful procedure and the cosmetic effects of the prick incision required to place the drainage catheter. However, with sufficient local anesthesia and the selection of an access path that is not along the neckline, these disadvantages can be minimized.

Microbiological assays of the material collected from USguided aspirations identified Staphylococcus aureus (79\%) as the predominant bacterial isolate present in cases of mastitis puerperalis. For cases of non-puerperal mastitis, a broad spectrum of pathogens was detected, although $S$. aureus was again the most prominent pathogen detected. For cases involving other forms of inflammation (i.e. folliculitides or infected seromas), $57 \%$ of cases were negative for germ growth. Therefore, based on the pathogens identified, the administration of a penicillinase-resistant antibiotic for cases of puerperal and non-puerperal mastitis would be recommended.

In addition to microbiological examination, all material collected from US-guided aspirations from patients with nonpuerperal mastitis were also examined cytopathologically. In 1 case, the cytopathological analysis provided a diagnosis of invasive ductal carcinoma of the breast in a postmenopausal patient. This case highlights the need for mastitis of unknown etiology, particularly of the non-puerperal type, to be examined histologically or cytologically to check for malignancy. Correspondingly, an excision biopsy should be performed during surgical therapy, or imaging should be performed following a course of conservative therapy.

When non-puerperal mastitis is diagnosed in older women, it is important to determine whether it represents an inflam- matory carcinoma, which is a breast cancer associated with an extremely high rate of morbidity and mortality. However, differentiating inflammatory carcinomas from acute mastitis can be difficult. Inflammatory breast cancer typically occurs in older women, while acute mastitis usually affects younger, lactating women. Therefore, if a trial of antibiotics does not decrease the signs and symptoms in the inflamed breast, especially in older, non-lactating women, inflammatory breast cancer must be considered [10]. Contrast-enhanced MRI of the breast can be performed after treatment for mastitis in order to determine whether a course of antibiotics was successful and to detect whether a histologically unconfirmed inflammatory carcinoma may be present [11]. In certain cases, MRI of the breast may be a reasonable addition to a US examination of the breast performed in the acute phase of a diagnosis, although a mammography (MG) should not be performed while the breast is inflamed. The compression of the breast required for $\mathrm{MG}$ increases the risk of rupturing the abscess collection, let alone the pain associated with pressure on the inflamed region. However, at our institution, it is common for an MG to be performed once the inflammation associated with non-puerperal mastitis subsides, in order to exclude the presence of a carcinoma. In this study, 109/110 patients with suspected abscesses were examined by US for diagnosis. In 4 cases, dynamic contrast-enhanced MRI of the breast was also performed, which has the advantage of being able to detect deep abscesses. Furthermore, the extent of the perifocal edema zone, which in most cases corresponds to the region of phlegmonous reaction in the surrounding tissue, can be easily detected, thereby allowing the portion of tissue that will respond well and quickly to antibiotics to be identified. However, the disadvantages of contrast-enhanced MR examinations are their limited availability and cost, making it a technique reserved for only select cases, e.g. those with deep lesions in hyperplastic breasts or in complicated cases.

The results from this study indicate that larger cavities in the mammae (e.g. due to fibrocystic alteration or galactostasis) in the presence of previously damaged breast tissue (e.g. due to previous surgeries or radiation therapy) promote the occurrence of an abscessing inflammation in the breast. Additional risk factors that have been associated with breast abscesses include smoking and DM [12-14]. With a reported smoker rate of only $18 \%$ among the enrolled patients, adequate documentation of nicotine abuse for all of the enrolled patients in this retrospective study was unlikely and therefore was not evaluated as a risk factor. Similarly, insufficient data to assess the role of DM as a risk factor for breast abscess also occurred so that DM could not be evaluated in this study. These latter 2 examples highlight the strengths and weaknesses of this study in that a large patient pool was able to be enrolled, yet incomplete patient data was obtained, which can be a common challenge in retrospective studies. 


\section{Conclusions}

The results of this study indicate that US-guided minimally invasive therapy, in combination with antibiotics, can be a successful strategy for the treatment of breast abscesses in a multi-modality breast team approach. For cases where a larger abscess is involved, a minimally invasive approach can still be maintained with the insertion of an additional drainage catheter to provide effective treatment.

\section{Disclosure Statement}

The authors declare no conflicts of interests.

\section{References}

1 Hayes R, Michell M, Nunnerley HB: Acute inflammation of the breast - the role of breast ultrasound in diagnosis and management. Clin Radiol 1991;44:253-256.

2 Jackson VP: The role of US in breast imaging. Radiology 1990;177:305-311.

3 Cunningham FG, MacDonald PC, Gant NF, et al. (eds): Williams Obstetrics, ed 20. Stamford, Appleton \& Lange, 1997, pp 564-565.

4 Strauss A, Heer IM, Müller-Egloff S, et al.: Breast abscess - A change of therapeutic standards. Senologie 2006;3:57-64.

5 Elagili F, Abdullah N, Fong L, Pei T: Aspiration of breast abscess under ultrasound guidance: Outcome obtained and factors affecting success. Asian J Surg 2007;30:40-44.
6 Rageth CJ, Ricklin ES, Scholl B, et al.: Conservative treatment of puerperal breast abscesses with repeated sonographically guided aspirations and oral antibiotic administrations. Z Geburtshilfe Neonatol 2004;208:170-173.

7 Schwarz RJ, Shrestha R: Needle aspiration of breast abscesses. Am J Surg 2001;182:117-119.

8 Christensen AF, Al-Suliman N, Nielsen KR, et al.: Ultrasound-guided drainage of breast abscesses: results in 151 patients. Br J Radiol 2005;78:186188.

9 Harish SK: The catheter drainage of breast abscesses: Is it going to be the future treatment of choice for puerperal breast abscess disease? Breast J 1997:3:357-359.

10 Dahlbeck SW, Donnelly JF, Theriault RL: Differentiating inflammatory breast cancer from acute mastitis. Am Fam Physician 1995;52:929-934.
1 Tomczak R, Rieber A, Zeitler H, et al.: The value of MR-mammography at 1.5 Tesla in the differential diagnosis of non-puerperal mastitis and inflammatory breast carcinoma. Rofo 1996;165:148-151.

12 Bharat A, Gao F, Aft RL, et al.: Predictors of primary breast abscesses and recurrence. World J Surg 2009;33:2582-2586.

13 Salmon RJ: Recurrent breast abscess: Role of smoking. J Gynecol Obstet Biol Reprod 1996;25:242-243.

14 Rizzo M, Peng L, Frisch A, et al.: Breast abscesses in nonlactating women with diabetes: clinical features and outcome. Am J Med Sci 2009;338:123126. 